\title{
Identifying the Strength Grade for Finger Jointed Timber Species According to BS 5268-2:2002
}

\author{
C.K. Muthumala ${ }^{1 *}$, S. De Silva ${ }^{2}$, K.K.I.U. Arunakumara ${ }^{3}$ and P.L.A.G. Alwis ${ }^{4}$ \\ ${ }^{1}$ Research, Development and Training Division, State Timber Corporation, Battaramulla, Sri Lanka \\ ${ }^{2}$ Department. of Civil and Environmental Engineering, Faculty of Engineering, \\ University of Ruhuna, Hapugala, Sri Lanka \\ ${ }^{3}$ Department of Crop Science, Faculty of Agriculture, University of Ruhuna, Kamburupitiya, Sri Lanka \\ ${ }^{4}$ Department of Agricultural Engineering, Faculty of Agriculture, University of Ruhuna, Kamburupitiya,
} Sri Lanka

Date Received 22-11-2018

Date Accepted 24-12-2019

\begin{abstract}
Off-cut wood is currently considered as waste thus is dumped by saw mills as they find no means of utilising them. Sawn timber material of furniture factories and short length of sawn timbers are also considered to be wastes in the timber industry. Finger jointing technique which interlock the end joints formed by machining a number of similar tapered symmetrical fingers are recognised in this regards as effective and sustainable means of utilisation of timber wastes. The present study was undertaken to assess the strength grade of finger jointed timber based on BS 5268-2:2002. Seven timber species which are commonly used in Sri Lanka were employed for the assessment with and without finger joints. Values of Modulus of Rupture (MOR), Modulus of Elasticity (MOE), Compression parallel to grain and Compression perpendicular to grain strength of the specimens were measured. Finger jointing was performed with constant geometry (finger length $19 \mathrm{~mm}$, tip width $1 \mathrm{~mm}$ and finger pitch $4 \mathrm{~mm}$ ) using polyvinyl acetate (PVA, P-SWR) adhesive at normal exposure conditions. The strength properties were evaluated by Universal Testing Machine (UTM) according to BS 373:1957. Strength classes relevant to the grade stresses were not significant for finger jointed and clear specimens of Satin, Mahogany, Jack and Grandis. Both clear and finger jointed timber specimens obtained D40 for Satin and Teak, D30 for Jack, Mahogany and Grandis. Teak shows properties similar to both D35 and D40 when used as finger jointed timber. Kumbuk was shown to change from D40 to D30 while using as finger jointed timber. Finger jointed Pine showed stress grade of C22, C24 and C27.
\end{abstract}

Keywords: Finger joint; grade stress, universal testing machine

\section{Introduction}

Timber is considered to be one of the oldest building materials used in Sri Lanka. It is widely used as a structural element in the construction industries thus has a steadily increased demand. In Sri Lanka there are over 400 different timber species (Muthumala and Amarasekara, 2013). During the processing, a considerable portion of timber is removed as waste dumped by sawmills. Dumping of timber as wastes and shorter sections is becoming a matter of great concern due to its scarcity. Therefore, innovative means of timber utilisation in particular, making use of waste sawn timber planks receives high recognition (Muthumala et al, 2018). A substantial portion of off-cut timbers in Sri Lanka is dumped as unused timber. No effective means has so far been found for the effective utilisation of off-cut timbers. Even though, a

*Correspondence: ck_muthumala@yahoo.com

Tel: +94777834716

ISSN 2235-9370 Print/ISSN 2235-9362 Online (C) University of Sri Jayewardenepura 55 
number of woodworking joints is employed for the utilisation of large timber structural elements, use of small timber sections for structural purposes is yet to be explored.

If the off-cut timber could be used for making such structural elements, timber wastage could substantially be minimised. Finger joint is considered to be a sustainable, eco-friendly and economically viable technique which ensures sustainable utilisation of small timber planks (Sandika et al, 2017).

Finger joints are described as interlocking end joints formed by machining a number of similar tapered symmetrical fingers at their ends using finger joint cutters and then binding together (BSI, BS EN 15497, 2014). Finger joints with off-cut timber may be useful in obtaining compression member with higher cross sections. As well as, production of timbers with high aesthetical value for different species is also ensured through this technique. The defects commonly found in the timbers could also be removed with this technique.

Even though the strength of finger jointed timber sections of softwoods has been evaluated extensively, little attention has so far been made on the hardwoods. However, most of the commonly used timber species in Sri Lanka are hardwoods. Therefore, the technology required for making finger joints with hardwoods has been developed and made available for local use. Finger length of $19 \mathrm{~mm}$ and polyvinyl acetate SWR adhesive was found to be the most suitable combination for making finger joints for local timber species (Muthumala et al., 2018). Furthermore the combination is recommended for the hardwoods and hardwood off-cuts which are used as structural components in the construction industry in Sri Lanka. In the future, fingerjointed material is certain to become more common place in both lumber and finished products as a means of upgrading defective material to clear and in creating otherwise unavailable long lengths (Hoadley, 2000).

Despite the fact that the structural performance of the finger jointed timber products such as studs, trusses, columns, beams etc., has been investigated the connections properties of finger joints have been investigated thus not included in the British Standards (BS) design code. Therefor the main objective of this study is to assess the strength grade of finger jointed timber based on BS 5268-2:2002. Which provides with necessary guidance on the structural use of timber, glued laminated timber, plywood, tempered hardboard and wood particle boards in load bearing members. It includes the recommendations on the quality, grade stresses and modification factors applicable to these materials when they are used as simple members, or as parts of built-up components, or as parts of structural components with the incorporation of other materials.

In literature, strength evaluation of finger jointed sections has been mainly found to be concentrated on temperate species (Fisette and Rice 1988, Samson 1985). As far as hardwoods are concerned, there are some reports from Ghana (Ayarkwa et al. 2000). A recent report from India tries to explain the measured flexural parameters of Eucalyptus in terms of the joint areas (Kumar et al. 2013).

\section{Methodology}

\subsection{Sample collection}

Six timber species namely; Grandis (Eucalyptus grandis), Jack (Aartocarpus heterphyllus), Kumbuk (Terminelia arjuna), Mahogany (Swietenia macrophylla), Satin (Chloroxylon swietenia) and Teak (Tectona grandis), were selected as hardwoods and Pine (Pinus caribaea), was selected as softwood considering their very high usage in Sri Lanka. Timber materials labelled as wastes were collected from the State Timber Corporation (STC) saw mill at Boossa, Sri Lanka and used for the 
study. The timber sections were selected from visually inspected defects free portions before been further treatments.

\subsection{Specimen Preparation}

Specimens were prepared using seasoned (M.C. 12\%) timber planks according to BS standards (BS 373:1957 and BS EN 15497:2014). Ten clear timber specimens were used as control specimens and 10 finger jointed timber specimens with same dimensions were made with constant finger geometry (finger length $19 \mathrm{~mm}$, tip width $1 \mathrm{~mm}$ and finger pitch $4 \mathrm{~mm}$ ) as shown in Figure 1. Specimens of clear and finger jointed were prepared from two timber planks with five replicates each.

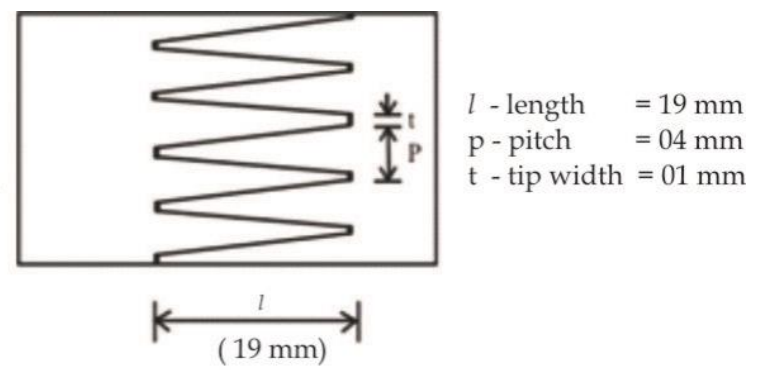

Figure 1. Finger geometry of a finger joint.

Finger jointing was performed on mid span, polyvinyl acetate- SWR adhesive was used for gluing at normal exposure conditions (Vievek et al, 2016). Specimens were prepared at the furniture factory of the State Timber Corporation, Boossa, Galle, Sri Lanka.

\subsection{Specimen for three-point bending test (MOR and MOE)}

The off-cut woods were dried using a kiln drier to ensure the wood moisture content is below $12 \%$. The average moisture content of wood materials was determined using a moisture meter before the preparation of test specimens. The samples were tested based on B.S. 373:1957 standards.

\subsection{Finger joint preparation}

The specimens with the dimension of $300 \mathrm{~mm} \times 25 \mathrm{~mm} \times 25 \mathrm{~mm}$ (moisture content of 12\%) were cross cut into two pieces using a circular saw and used for the static bending tests (the length of a piece was $160 \mathrm{~mm}$ ). The specimens with the dimension of $60 \mathrm{~mm} \times 20 \mathrm{~mm} \times 20 \mathrm{~mm}$ were cross cut into two pieces using the same saw machine and used for the compression test-parallel to grains. Another set of specimen with the dimension of $50 \mathrm{~mm} \times 50 \mathrm{~mm} \times 50 \mathrm{~mm}$ were cross cut into two pieces and used for the compression test-perpendicular to grains (BS 373: 1957).

\subsection{Experimental procedure}

Sample testing was performed at the laboratory of STC, Battaramulla, Colombo, Sri Lanka. Three-point bending test and compression tests of the prepared specimens were conducted using Universal Testing Machine (UTM) (give the type/brand, country of produced etc) according to BS 373:1957. Before loading by UTM, the average density and natural moisture content were measured for each species.

\subsection{Average density}

The timber samples were placed in an oven at $105^{\circ} \mathrm{C}$ for 48 hours and the dry weight was measured. The density values were calculated for the seven timber species using equation 1). (BS EN 373:1957). 
Density $=\frac{\text { Weight of oven dry wood }(\mathrm{kg})}{\text { Volume of wood }\left(\mathrm{m}^{3}\right)}$

Determination of basic density was done based on the green volume and oven-dry weight using a water displacement method. Samples placed at room temperature showed good structural performance compared to those placed at hot and wet conditioned (give details) as demonstrated by Vievek et al, (2016).

\subsection{Bending test}

Specimens were tested by three-point bending test to obtain bending strength. The span was $280 \mathrm{~mm}$ for the test and load was applied on mid span of the specimen at a loading speed of 6 $\mathrm{mm} / \mathrm{min}$. Displacement was recorded with the applied load and load vs displacement graph was plotted.

Modulus of Rupture (MOR) was calculated for the ultimate state using load vs displacement graph. Maximum load represented the ultimate load and the maximum load in elastic region represented the serviceability load. When the applied load is the ultimate load, bending strength is taken as Modulus of Rupture.

\subsection{Modulus of elasticity (MOE)}

Modulus of Elasticity is considered to be an indicator for stiffness of the wood and only applies to the conditions within the elastic limit. It is the ratio of stress per unit area to the deformation per unit length. Flexural test resulted at serviceability state was used to obtain the MOE in this study.

\subsection{Compression parallel to grain test}

Compression parallel to grain test was carried out with loading plate moving speed of 0.5 $\mathrm{mm} \mathrm{min}^{-1}$ and load vs. displacement variation was obtained. Compressive strength of clear timber at ultimate state was calculated by the maximum load acting on the timber before the failure which was obtained from the load deflection curve of compression parallel to grain test. Maximum load of the elastic limit was used to obtain the serviceability state compressive strength.

\subsection{Compression perpendicular to grain test}

Failure of the specimens was determined by loading them perpendicular to grain with loading plate moving speed of $0.5 \mathrm{~mm} \mathrm{~min}^{-1}$. Displacement was obtained with the load applied and load vs. displacement curve was plotted. The maximum load identified with the graph was used to calculate ultimate compressive strength and the maximum load of the elastic region was used to calculate serviceability compressive strength perpendicular to grain.

\subsection{Strength class identification from BS 5268-2:2002}

Strength classes appeared in BS 5268-2:2002 are relevant to the grade stresses of timber at serviceability state. Therefore, the characteristic strength obtained from the bending and compression test were employed to derive grade stresses.

\subsection{Grade stress-bending}

Characteristic bending strength was calculated by the following factors to derive grade bending stresses. According to BS5268-2; Section depth less than $72 \mathrm{~mm}-0.856$, Duration of the load very short term- 0.571

Grade stress $=$ Strength in Serviceability Limit State $\left(\mathrm{N} \mathrm{mm}^{-2}\right) \times 0.856 \times 0.571$ 


\section{Results}

As shown in Table 1, the average density values varied with the species and ranged between 440$980 \mathrm{~kg} \mathrm{~m}^{-3}$. The highest average density value shows in Satin timber species and the least average density value shows in Pine timber species.

Table 1: Average density and timber class of selected timber species.

\begin{tabular}{lcl}
\hline Species & $\begin{array}{c}\text { Average density } \\
\left(\mathrm{kg} \mathrm{m}^{-3}\right)\end{array}$ & $\begin{array}{l}\text { Timber class according to } \\
\text { STC classification }\end{array}$ \\
\hline Teak & 740 & Super Luxury \\
Satin & 980 & Luxury \\
Mahogany & 560 & Luxury \\
Jack & 640 & Luxury \\
Kumbuk & 720 & Special Class \\
Grandis & 550 & Class II \\
Pine & 440 & Class III \\
\hline
\end{tabular}

According to BS 373:1957, the average moisture content of the specimen should approximately be $12 \%$ at normal exposure conditions for testing. As depicted in Table 2, the moisture contents varied within $9.38-13.83 \%$ thus, declared a suitable timber specimens for testing.

Table 2: Average moisture content of tested specimen.

\begin{tabular}{lccccrc}
\hline Species & \multicolumn{2}{c}{$\begin{array}{c}\text { Specimen used for bending } \\
\text { test }(\%)\end{array}$} & \multicolumn{2}{c}{$\begin{array}{c}\text { Specimen used for compression } \\
\text { parallel to grain test }(\%)\end{array}$} & \multicolumn{2}{c}{$\begin{array}{c}\text { Specimen used for compression } \\
\text { perpendicular to grain test }(\%)\end{array}$} \\
\hline & Clear & FJ & Clear & FJ & Clear & FJ \\
\hline Teak & 10.50 & 10.28 & 10.63 & 10.58 & 9.98 & 10.05 \\
Satin & 12.13 & 12.60 & 11.15 & 11.10 & 10.55 & 10.50 \\
Mahogany & 10.85 & 10.88 & 10.35 & 10.33 & 9.68 & 10.03 \\
Jack & 12.33 & 12.50 & 9.38 & 9.58 & 10.70 & 10.65 \\
Kumbuk & 11.53 & 11.15 & 11.30 & 12.18 & 13.83 & 13.30 \\
Grandis & 13.50 & 13.35 & 11.35 & 11.65 & 12.45 & 12.10 \\
Pine & 11.35 & 11.38 & 11.15 & 11.60 & 10.43 & 10.40 \\
\hline
\end{tabular}

Table 3 shows that the characteristic bending strength reduction is less for Satin and it is $9.5 \%$ compared to clear timber. Teak and Pine also have strength reduction $\%$ less than $20 \%$. Higher strength reduction \% is shows in Kumbuk timber species.

Table 3: Average serviceability bending strength of tested specimen.

\begin{tabular}{lccc}
\hline Species & $\begin{array}{c}\text { Clear timber } \\
\text { section }\left(\mathrm{N} \mathrm{mm}^{-2}\right)\end{array}$ & $\begin{array}{c}\text { Finger jointed timber } \\
\text { section }\left(\mathrm{N} \mathrm{mm}^{-2}\right)\end{array}$ & $\begin{array}{c}\text { Strength reduction } \\
\text { percentage } \%\end{array}$ \\
\hline Teak & 26.02 & 23.20 & 10.84 \\
Satin & 27.94 & 25.28 & 9.50 \\
Mahogany & 24.59 & 16.64 & 32.34 \\
Jack & 30.58 & 17.49 & 42.82 \\
Kumbuk & 25.77 & 13.26 & 48.54 \\
Grandis & 29.39 & 16.09 & 45.25 \\
Pine & 20.86 & 16.80 & 19.43 \\
\hline
\end{tabular}

According to Table $4 \mathrm{MOE}$ for clear timber and finger jointed timber are approximately same and Pine (soft wood) shows MOE increment and other species have MOE reduction less than $20 \%$. The highest MOE reduction \% is shows in Kumbuk timber species. 
Table 4: Average MOE for tested specimens.

\begin{tabular}{lccc}
\hline Species & $\begin{array}{c}\text { Clear timber specimen } \\
\left(\mathrm{N} \mathrm{mm}^{-2}\right)\end{array}$ & $\begin{array}{c}\text { Finger jointed timber } \\
\text { specimen }\left(\mathrm{N} \mathrm{mm}^{-2}\right)\end{array}$ & $\begin{array}{c}\text { MOE reduction } \\
\text { percentage\% }\end{array}$ \\
\hline Teak & 8865.07 & 8796.66 & 0.77 \\
Satin & 9703.65 & 9493.32 & 2.17 \\
Mahogany & 6208.59 & 5552.56 & 10.57 \\
Jack & 5537.37 & 5391.96 & 2.63 \\
Kumbuk & 5225.88 & 4383.83 & 16.11 \\
Grandis & 5375.64 & 5286.38 & 1.66 \\
Pine & 5361.99 & 6657.08 & -24.15 \\
\hline
\end{tabular}

Serviceability Compressive strength parallel to grain of Jack is almost similar for clear and finger jointed timber according to Table 5. Satin, Mahogany, Grandis and Pine also have strength reduction less than $20 \%$ at serviceability state. The highest Average compressive strength parallel to grain reduction $\%$ is shows in Kumbuk timber species.

Table 5: Average compressive strength parallel to grain for tested specimens at Serviceability state.

\begin{tabular}{lccc}
\hline Species & $\begin{array}{c}\text { Clear timber } \\
\text { section }\left(\mathrm{N} \mathrm{mm}^{-2}\right)\end{array}$ & $\begin{array}{c}\text { Finger jointed timber } \\
\text { section }\left(\mathrm{N} \mathrm{mm}^{-2}\right)\end{array}$ & $\begin{array}{c}\text { Strength reduction } \\
\text { percentage \% }\end{array}$ \\
\hline Teak & 24.45 & 18.20 & 25.54 \\
Satin & 42.21 & 36.62 & 13.24 \\
Mahogany & 15.62 & 13.51 & 13.51 \\
Jack & 14.93 & 14.70 & 1.53 \\
Kumbuk & 29.53 & 20.17 & 31.68 \\
Grandis & 15.61 & 13.55 & 13.22 \\
Pine & 15.89 & 15.40 & 3.04 \\
\hline
\end{tabular}

Table 6 depicts that compression perpendicular to grain test significantly different from bending and compression test results because finger jointed timber strengths have been increased for all the specimens other than Jack compared to clear timber.

Table 6: Average compressive strength perpendicular to grain for tested specimens at serviceability state.

\begin{tabular}{lccc}
\hline Species & $\begin{array}{c}\text { Clear timber } \\
\text { section }\left(\mathrm{N} \mathrm{mm}^{-2}\right)\end{array}$ & $\begin{array}{c}\text { Finger jointed timber } \\
\text { section }\left(\mathrm{N} \mathrm{mm}^{-2}\right)\end{array}$ & $\begin{array}{c}\text { Strength } \\
\text { reduction \% }\end{array}$ \\
\hline Teak & 8.53 & 10.08 & -18.13 \\
Satin & 15.51 & 17.16 & -10.66 \\
Mahogany & 7.85 & 8.13 & -3.66 \\
Jack & 13.43 & 11.03 & 17.90 \\
Kumbuk & 7.71 & 8.28 & -7.31 \\
Grandis & 5.14 & 5.38 & -4.72 \\
Pine & 6.06 & 7.72 & -27.39 \\
\hline
\end{tabular}

Grade Stress values of Table 7 were calculated by Bending Strength in Serviceability state $\times 0.856 \times 0.571$. 
Table 7: Grade Bending Stresses for Clear and Finger Jointed specimens.

\begin{tabular}{lcc}
\hline Species & $\begin{array}{c}\text { Clear Timber } \\
\text { Section }\left(\mathrm{N} \mathrm{mm}^{-2}\right)\end{array}$ & $\begin{array}{c}\text { Finger Jointed Timber } \\
\text { Section }\left(\mathrm{N} \mathrm{mm}^{-2}\right)\end{array}$ \\
\hline Teak & 12.71 & 11.33 \\
Satin & 13.64 & 12.35 \\
Mahogany & 12.01 & 8.13 \\
Jack & 14.94 & 8.54 \\
Kumbuk & 12.59 & 6.48 \\
Grandis & 14.35 & 7.86 \\
Pine & 10.19 & 8.21 \\
\hline
\end{tabular}

In Table 8, average grade stresses for compression parallel to grain values were obtained according to BS 5268-2, considering very short term for the duration of the load characteristic strength should be multiplied by 0.571 .

Table 8: Average grade stresses for compression parallel to grain test.

\begin{tabular}{lcc}
\hline Species & $\begin{array}{c}\text { Clear Timber } \\
\text { Specimen }\left(\mathrm{N} / \mathrm{mm}^{2}\right)\end{array}$ & $\begin{array}{c}\text { Finger Jointed Timber } \\
\text { Specimen }\left(\mathrm{N} / \mathrm{mm}^{2}\right)\end{array}$ \\
\hline Teak & 13.97 & 10.40 \\
Satin & 24.12 & 20.93 \\
Mahogany & 8.93 & 7.72 \\
Jack & 8.53 & 8.40 \\
Kumbuk & 16.87 & 11.53 \\
Grandis & 8.92 & 7.74 \\
Pine & 9.08 & 8.80 \\
\hline
\end{tabular}

In table 9, average grade stresses for compression perpendicular to grain values were obtained according to BS 5268-2, considering very short term for the duration of the load characteristic strength should be multiplied by 0.571 .

Table 9: Average grade stresses for compression perpendicular to grain test.

\begin{tabular}{lcc}
\hline Species & $\begin{array}{c}\text { Clear Timber } \\
\text { Specimen }\left(\mathrm{N} \mathrm{mm}^{-2}\right)\end{array}$ & $\begin{array}{c}\text { Finger Jointed Timber } \\
\text { Specimen }\left(\mathrm{N} \mathrm{mm}^{-2}\right)\end{array}$ \\
\hline Teak & 4.88 & 5.76 \\
Satin & 8.86 & 9.80 \\
Mahogany & 4.48 & 4.65 \\
Jack & 7.67 & 6.30 \\
Kumbuk & 4.41 & 4.73 \\
Grandis & 2.94 & 3.07 \\
Pine & 3.46 & 4.41 \\
\hline
\end{tabular}

Table 10 and Table 11 show that Grade Stress values are derived using lower and higher values of BS 5268-2:2002. According to the calculation in Table 10 and 11, strength classes are included in Table 12 . 
Table 10: Grade strength comparison for clear timber according to BS 5268-2:2002.

\begin{tabular}{|c|c|c|c|c|c|c|c|c|c|c|c|c|c|c|c|c|c|c|c|c|c|}
\hline \multirow{3}{*}{ Clear Timber } & \multicolumn{18}{|c|}{ HARDWOOD } & \multirow{2}{*}{\multicolumn{3}{|c|}{$\begin{array}{c}\text { SOFT WOOD } \\
\text { Pine }\end{array}$}} \\
\hline & \multicolumn{3}{|c|}{ Teak } & \multicolumn{3}{|c|}{ Satin } & \multicolumn{3}{|c|}{ Mahogany } & \multicolumn{3}{|c|}{ Jak } & \multicolumn{3}{|c|}{ Kumbuk } & \multicolumn{3}{|c|}{ Grandis } & & & \\
\hline & Lower & Test & Higher & Lower & Test & Higher & Lower & Test & Higher & Lower & Test & Higher & Lower & Test & Higher & Lower & Test & Higher & Lower & Test & Higher \\
\hline \multirow{3}{*}{ Bending } & 12.5 & 12.71 & 16 & 12.5 & 13.64 & 16 & 11 & 12.01 & 12.5 & 12.5 & 14.94 & 16 & 12.5 & 12.59 & 16 & 12.5 & 14.35 & 16 & 10 & 10.19 & 11 \\
\hline & $1.652 \%$ & & $-25.885 \%$ & $8.358 \%$ & & $-17.302 \%$ & $8.410 \%$ & & $-4.080 \%$ & $16.332 \%$ & & $-7.095 \%$ & $0.715 \%$ & & $-27.085 \%$ & $12.892 \%$ & & $-11.498 \%$ & $1.865 \%$ & & $-7.949 \%$ \\
\hline & D40 & D40 & D50 & D40 & D40 & D50 & D35 & D40 & D40 & D40 & D50 & D50 & D 40 & D40 & D50 & D 40 & D50 & D50 & $\mathrm{C} 27$ & $\mathrm{C} 27$ & $\mathrm{C} 30$ \\
\hline & & & & & & & & & & & & & & & & & & & & & \\
\hline \multirow{3}{*}{ MOE(Mean) } & & 8865.1 & 9500 & 9500 & 9703.65 & 10000 & & 6208.6 & 9500 & & 5537.37 & 9500 & & 5225.9 & 9500 & & 5375.64 & 9500 & & 5361.99 & 6800 \\
\hline & & & $-7.162 \%$ & $2.099 \%$ & & $-3.054 \%$ & & & $-53.014 \%$ & & & $-71.562 \%$ & & & $.81 .788 \%$ & & & $.76 .723 \%$ & & & $-26.819 \%$ \\
\hline & & D30 & D30 & D30 & D30 & D35 & & D30 & D30 & & D30 & D30 & & D30 & D30 & & D30 & D30 & & $\mathrm{Cl} 4$ & $\mathrm{Cl} 4$ \\
\hline \multirow{3}{*}{ MOE(Minimum) } & 7500 & 8865.1 & 12600 & 7500 & 9703.65 & 12600 & 6000 & 6208.6 & 6500 & & 5537.37 & 6000 & & 5225.9 & 6000 & & 5375.64 & 6000 & 4600 & 5361.99 & 5800 \\
\hline & $15.398 \%$ & & $-42.131 \%$ & $22.709 \%$ & & $-29.848 \%$ & $3.360 \%$ & & $-4.694 \%$ & & & $-8.355 \%$ & & & $-14.813 \%$ & & & $-11.615 \%$ & $14.211 \%$ & & $-8.169 \%$ \\
\hline & D40 & D40 & D50 & D40 & D40 & D50 & D30 & $\mathrm{D} 30$ & D35 & & D30 & D30 & & $\mathrm{D} 30$ & D30 & & D30 & D30 & $\mathrm{Cl4}$ & $\mathrm{C} 16$ & $\mathrm{C} 16$ \\
\hline & & & & & & & & & & & & & & & & & & & & & \\
\hline \multirow{3}{*}{ Compression // } & 12.6 & 13.97 & 15.2 & 23 & 24.12 & & 8.6 & 8.93 & 12.6 & 8.1 & 8.53 & 8.6 & 15.2 & \begin{tabular}{|l|}
16.87 \\
\end{tabular} & 18 & 8.6 & 8.92 & 12.6 & 8.7 & 9.08 & \\
\hline & $9.807 \%$ & & $-8.805 \%$ & \begin{tabular}{|l|l|}
$4.643 \%$ \\
\end{tabular} & & & $3.695 \%$ & & $-41.097 \%$ & $5.041 \%$ & & $-0.821 \%$ & 9.899\% & & $-6.698 \%$ & $3.587 \%$ & & $-41.256 \%$ & $4.185 \%$ & & \\
\hline & D40 & D50 & D50 & D70 & D70 & & D35 & D35 & D40 & D30 & D35 & D35 & D50 & $\mathrm{D} 60$ & D60 & D35 & D35 & D40 & $C 40$ & $C 40$ & \\
\hline & & & & & & & & & & & & & & & & & & & & & \\
\hline \multirow{3}{*}{ Compression 1} & 4.6 & 4.88 & & 4.6 & 8.86 & & 4 & 4.48 & 4.6 & 4.6 & 7.67 & & 4 & 4.41 & 4.6 & 2.6 & 2.94 & 3 & 2.6 & 3.46 & \\
\hline & $5.738 \%$ & & & $48.081 \%$ & & & $10.714 \%$ & & $-2.679 \%$ & $40.026 \%$ & & & $9.297 \%$ & & $-4.308 \%$ & $11.565 \%$ & & $-2.041 \%$ & $24.855 \%$ & & \\
\hline & $\mathrm{D} 70$ & D70 & & D70 & D70 & & D60 & D70 & D70 & $\mathrm{D} 70$ & $\mathrm{D} 70$ & & D60 & $\mathrm{D} 70$ & D70 & D35 & D40 & D40 & $C 40$ & $C 40$ & \\
\hline & & & & & & & & & & & & & & & & & & & & & \\
\hline \multirow{3}{*}{ Average Density } & 700 & 740 & 780 & 840 & 980 & 1080 & & 560 & 640 & & 640 & & 700 & 720 & 780 & & 550 & 640 & 420 & 440 & 450 \\
\hline & $5.405 \%$ & & \begin{tabular}{|l|}
$-5.405 \%$ \\
\end{tabular} & $14.286 \%$ & & $-10.204 \%$ & & & $-14.286 \%$ & & & & $2.778 \%$ & & $-8.333 \%$ & & & \begin{tabular}{|l|}
$-16.364 \%$ \\
\end{tabular} & $4.545 \%$ & & $-2.273 \%$ \\
\hline & D40 & D40 & D50 & D60 & D70 & D70 & & D30 & D30 & & D30 & & D40 & D40 & D50 & & D30 & D30 & $\mathrm{C} 24$ & $\mathrm{C} 27$ & $\mathrm{C} 27$ \\
\hline \multirow{2}{*}{\multicolumn{2}{|c|}{ Overall strength class }} & & & & & & & & & & & & & & & & & & & & \\
\hline & & D40 & & & D70D40 & & & D30 & & & D30 & & & D40 & & & D30 & & & $\mathrm{C} 27$ & \\
\hline
\end{tabular}

Table 11: Grade strength comparison for finger jointed timber according to BS 5268-2:2002.

\begin{tabular}{|c|c|c|c|c|c|c|c|c|c|c|c|c|c|c|c|c|c|c|c|c|c|}
\hline \multirow{3}{*}{ FJ Timber } & \multicolumn{18}{|c|}{ HARDWOOD } & \multirow{2}{*}{\multicolumn{3}{|c|}{$\begin{array}{c}\text { SOFT WOOD } \\
\text { Pine }\end{array}$}} \\
\hline & \multicolumn{3}{|c|}{ Teak } & \multicolumn{3}{|c|}{ Satin } & \multicolumn{3}{|c|}{ Mahogany } & \multicolumn{3}{|c|}{ Jak } & \multicolumn{3}{|c|}{ Kumbuk } & \multicolumn{3}{|c|}{ Grandis } & & & \\
\hline & Lower & Test & Higher & Lower & Test & Higher I & Lorwer & Test & Higher & Lower & Test & Higher & Lower & Test & Higher & Lower & Test & Higher & Lower & Test & Higher \\
\hline \multirow{3}{*}{ Bending } & 11 & 11.33 & 12.5 & 12.5 & 12.35 & 16 & & 8.13 & 9 & & 8.54 & 9 & & 6.48 & 9 & & 7.86 & 9 & 7.5 & 8.21 & 10 \\
\hline & $2.913 \%$ & & $-10.327 \%$ & $\mid-1.215 \%$ & & $\mid-29.555 \%$ & & & $-10.701 \%$ & & & \begin{tabular}{|l|}
$.5 .386 \%$ \\
\end{tabular} & & & $-38.889 \%$ & & & $-14.504 \%$ & \begin{tabular}{|l|}
$8.648 \%$ \\
\end{tabular} & & $-21.803 \%$ \\
\hline & D35 & D35 & D40 & D40 & D40 & D50 & & D30 & D30 & & D30 & D30 & & D30 & D30 & & D30 & D30 & $\mathrm{C} 24$ & $\mathrm{C} 24$ & $\mathrm{C} 27$ \\
\hline & & & & & & & & & & & & & & & & & & & & & \\
\hline \multirow{3}{*}{$\operatorname{MOE}($ Mean $)$} & & 8796.7 & 9500 & & 9493.32 & 9500 & & 5552.6 & 9500 & & 5391.96 & 9500 & & 4383.8 & 9500 & & 5286.38 & 9500 & & 6657.08 & 6800 \\
\hline & & & $-7.996 \%$ & & & $-0.070 \%$ & & & \begin{tabular}{|l|}
$-71.092 \%$ \\
\end{tabular} & & & \begin{tabular}{|l|l|}
$.76 .188 \%$ \\
\end{tabular} & & & $-116.705 \%$ & & & \begin{tabular}{|l|}
$.79 .707 \%$ \\
\end{tabular} & & & $-2.147 \%$ \\
\hline & & D30 & D30 & & D30 & D30 & & D30 & D30 & & D30 & D30 & & D30 & D30 & & D30 & D30 & & $\mathrm{Cl} 4$ & $\mathrm{Cl} 4$ \\
\hline \multirow{3}{*}{ MOE(Minimum) } & 7500 & \begin{tabular}{|l|l}
8796.7 \\
\end{tabular} & 12600 & 7500 & 9493.32 & 12600 & & 5552.6 & 6000 & & 5391.96 & 6000 & & 4383.8 & 6000 & & 5286.38 & 6000 & 6500 & \begin{tabular}{|l|l|}
6657.08 \\
\end{tabular} & 7200 \\
\hline & $14.740 \%$ & & $-43.236 \%$ & $20.997 \%$ & & $-32.725 \%$ & & & $-8.058 \%$ & & & $\mid-11.277 \%$ & & & $-36.867 \%$ & & & $-13.499 \%$ & $2.360 \%$ & & $-8.156 \%$ \\
\hline & D40 & D40 & D50 & D40 & D40 & D50 & & $\mathrm{D} 30$ & D30 & & D30 & D30 & & D30 & D30 & & D30 & D30 & $\mathrm{C} 22$ & $\mathrm{C} 22$ & $\mathrm{C} 24$ \\
\hline & & & & & & & & & & & & & & & & & & & & & \\
\hline \multirow{3}{*}{ Compression // } & 8.6 & 10.4 & 12.6 & 18 & 20.93 & 23 & & 7.72 & 8.1 & 8.1 & 8.4 & 8.6 & 8.6 & \begin{tabular}{|l|}
11.53 \\
\end{tabular} & 12.6 & & 7.74 & 8.1 & 8.7 & 8.8 & \\
\hline & $17.308 \%$ & & $-21.154 \%$ & $13.999 \%$ & & $-9.890 \%$ & & & $-4.922 \%$ & $3.571 \%$ & & \begin{tabular}{|l|}
$-2.381 \%$ \\
\end{tabular} & $25.412 \%$ & & $-9.280 \%$ & & & $-4.651 \%$ & $1.136 \%$ & & \\
\hline & D35 & D35 & D40 & D60 & D70 & D70 & & $\mathrm{D} 30$ & D30 & D30 & D35 & D35 & D35 & D40 & D40 & & D35 & D30 & $C 40$ & $C 40$ & \\
\hline & & & & & & & & & & & & & & & & & & & & & \\
\hline \multirow{3}{*}{ Compression $\perp$} & 4.6 & 5.76 & & 4.6 & 9.8 & & 4.6 & 4.65 & & 4.6 & 6.3 & & 4.6 & 4.73 & & 3.5 & \begin{tabular}{|l|l}
3.07 \\
\end{tabular} & 4 & 2.6 & 4.41 & \\
\hline & $20.139 \%$ & & & $53.061 \%$ & & & $\mid 1.075 \%$ & & & $26.984 \%$ & & & $2.748 \%$ & & & $\mid-14.007 \%$ & & $-30.293 \%$ & $41.043 \%$ & & \\
\hline & D70 & D70 & & D70 & D70 & & D70 & $\mathrm{D} 70$ & & D70 & D70 & & D70 & $\mathrm{D} 70$ & & D50 & D50 & D60 & $\mathrm{C} 40$ & $C 40$ & \\
\hline \multicolumn{22}{|c|}{0} \\
\hline \multirow{3}{*}{ Average Density } & 700 & 740 & 780 & 840 & 980 & 1080 & & 560 & 640 & & 640 & & 700 & 720 & 780 & & 550 & 640 & 420 & 440 & 450 \\
\hline & $5.405 \%$ & & $-5.405 \%$ & $14.286 \%$ & & $-10.204 \%$ & & & $-14.286 \%$ & & & & $2.778 \%$ & & $-8.333 \%$ & & & $-16.364 \%$ & $4.545 \%$ & & $-2.273 \%$ \\
\hline & D40 & D40 & D50 & D60 & D70 & D70 & & $\mathrm{D} 30$ & D30 & & D30 & & D40 & D40 & D50 & & D30 & D30 & $\mathrm{C} 24$ & $\mathrm{C} 27$ & $\mathrm{C} 27$ \\
\hline \multicolumn{2}{|c|}{ Overall strength class } & D 40,35 & & & D70D40 & & & D30 & & & D30 & & & D 30 & & & D30 & & & $27 / \mathrm{C}_{4} / \mathrm{CP}^{2}$ & \\
\hline
\end{tabular}


Table 12: Strength class identified.

\begin{tabular}{llcc}
\hline Species & Category & Clear timber & $\begin{array}{c}\text { Finger jointed } \\
\text { timber }\end{array}$ \\
\hline Teak & Hardwood & D40 & D35/D40 \\
Satin & Hard wood & D40/D70 & D40/D70 \\
Mahogany & Hard wood & D30 & D30 \\
Jack & Hard wood & D30 & D30 \\
Kumbuk & Hard wood & D40 & D30 \\
Grandis & Hard wood & D30 & D30 \\
Pine & Soft wood & C27 & C22/24/27 \\
\hline
\end{tabular}

\subsection{Strength class for clear and finger jointed timber}

Strength class for selected timber species were obtained considering the average density, MOE and grade stresses included in Table 7, Table 8 and Table 9 which were obtained as experiment results. In this case, BS 5268-2:2002 was used and strength class for clear and finger jointed timber were obtained separately. Nearest higher and lower value for the test resulted value were obtained from BS 5268-2:2002 and considering the minimum deviation for grade stress which were obtained from test strength class were selected. Deviation was calculated as a percentage by using equation 3.

Deviation $=\frac{\text { Grade stress obtain as experiment results }- \text { Nearest grade stress }}{\text { Grade stress obtain as experiment results }} \times 100 \%$

\section{Discussion}

According to the BSI standard- BS 5268:2002, "Grade stresses for tropical hardwoods graded in accordance with BS 5756 rules for service classes 1 and 2", teak clear timber shows grade stresses for Bending-13.7 (N/mm²), Compression parallel to grain-13.4 $\left(\mathrm{N} \mathrm{mm}^{-2}\right)$, Compression perpendicular to grain-3.1 $\left(\mathrm{N} \mathrm{mm}^{-2}\right)$ and MOE- $7400\left(\mathrm{~N} \mathrm{~mm}^{-2}\right)$. In experimental results values are also closely similar to the standard values as Bending- $12.71\left(\mathrm{~N} \mathrm{~mm}^{-2}\right)$, Compression parallel to grain-13.97 $\left(\mathrm{N} \mathrm{mm}^{-2}\right)$, Compression perpendicular to grain-4.88 $\left(\mathrm{N} \mathrm{mm}^{-2}\right)$ and MOE- $8865\left(\mathrm{~N} \mathrm{~mm}^{-2}\right)$. So, accuracy of the Experimental values are acceptable.

According to Table 12, considering finger jointed timber, Teak shows similar to both D35 and D40. Finger jointed Satin, Mahogany, Jack and Grandis timber are almost similar to clear timber in this case. Kumbuk has been changed from D40 to D30 while use as finger jointed timber. (D represent-Hard wood and C represent-Soft wood). Pine clear timber shows C27 and Finger jointed Pine shows C22, C24 or C27. All the clear specimens and finger jointed specimens except Kumbuk, show nearly similar Grade stress classes. Thus, this finger joint technology can be used for producing elements.

\section{Conclusion}

Finger joint technique is used locally for manufacturing process in Sri Lanka considering the mechanical properties. Therefore, the reliability of finger joint as an alternative method of connecting timber structural elements needed to be evaluated. The performance of finger jointed standard specimens and the strength grade of them were studied based on BS 5268-2:2002.

No significant differences in strength classes relevant to the grade stresses were observed for finger jointed and clear specimens for Satin, Mahogany, Jack and Grandis. Both clear and finger jointed timber 
specimens obtained D40 for Satin and Teak, D30 for Jack, Mahogany and Grandis. Teak showed similar properties to both D35 and D40 when it was used as finger jointed timber.

Grade stress class was changed in Kumbuk species from D40 to D30 while it was used as finger jointed timber. Finger jointed Pine showed properties of C22, C24 and C27. The present findings proved that finger joint technique is useful in effective utilization of off-cut timbers to manufacture finger jointed structural and non-structural elements.

\section{Acknowledgment}

Authors thank to laboratory staff of the State Timber Corporation for their valuable assistants in research works.

\section{References}

Ayarkwa, J., Hirashima, Y. and Sasaki, Y., 2000. Effect of finger geometry and end pressure on the flexural properties of finger-jointed tropical African hardwoods. Forest Products Journal, 50:53-63.

British Standard Institution, 1999. BS 373: 1957. Methods of testing small clear specimens of timber. British Standards Institution. London.

British Standard Institution, 2014. BS EN 15497:2014, Structural finger jointed solid timber-performance requirements and minimum production requirements. European Committee for Standardisation. B 1000 Brussels.

British Standard Institution, 1996. BS EN 5268-2: 2002, Structural use of timber-Code of practise for permissible stress design, materials and workmanship. BSI. Chiswick High Road. London.

Fisette, P.R. and Rice, W.W., 1988. An analysis of structural finger-joints made from two north eastern species. Forest Products Journal, 38:40-44

Hoadley, R.B., 2000. Understanding wood: A craftsman's guide to wood technology, chapter 16, The Taunton press, USA. 241

Kumar, K., Sharma, V.S. and Gupta, C.M., 2013. Role of fingertip area on flexural strength properties of finger-jointed sections. International Wood Products Journal, 4:101-106.

Muthumala, C.K. and Amarasekara, H.S., 2013. Investigation the Authenticity of Local and Imported timber Species in Sri Lanka, Proceeding of the International Forestry and Environment Symposium, Department of Forestry and Environmental Science, University of Sri Jayewardenepura, 95.

Muthumala, C.K., De Siva, S. Alwis, P.L.A.G. and Aruna Kumara, K.K.I.U., 2018. Factors affecting the glue strength of finger-joints in commonly used timber species in Sri Lanka. International Symposium on Agriculture and Extension. Faculty of Agriculture. University of Ruhuna, Sri Lanka, 126-128.

Muthumala, C.K., De Silva, S., Alwis, P.L.A.G. and Arunakumara, K.K.I.U. Investigate the most suitable glue type for finger-joints production in Sri Lanka. Research Journal of Agriculture and Forestry Sciences, November, 2018. ISCA, India. 6:6-9.

Samson, M., 1985. Potential of finger-jointed lumber for machine stress-rated lumber grades. Forest Products Journal, 35:20-24.

Sandika, A.L., Pathirana, G.D.P.S. and Muthumala, C.K., 2017. Finger joint timber products for effective utilisation of natural resources: An analysis of physical properties. Economic factors and Consumers' perception. International Symposium on Agriculture and Environment, University of Ruhuna, Sri Lanka. 109-111.

Vievek, S., De Silva, S., De Silva, S. and Muthumala, C.K., 2016. Finger joint and their structural performance in different exposure conditions. $7^{\text {th }}$ International Conference on Sustainable Build Environment. Kandy. Sri Lanka, 207-210. 